\title{
Evolution of the TOR Pathway
}

\author{
Teunis J. P. van Dam • Fried J. T. Zwartkruis • \\ Johannes L. Bos • Berend Snel
}

Received: 23 June 2011/ Accepted: 24 October 2011/Published online: 5 November 2011

(C) The Author(s) 2011. This article is published with open access at Springerlink.com

\begin{abstract}
The TOR kinase is a major regulator of growth in eukaryotes. Many components of the TOR pathway are implicated in cancer and metabolic diseases in humans. Analysis of the evolution of TOR and its pathway may provide fundamental insight into the evolution of growth regulation in eukaryotes and provide a practical framework on which experimental evidence can be compared between species. Here we performed phylogenetic analyses on the components of the TOR pathway and determined their point of invention. We find that the two TOR complexes and a large part of the TOR pathway originated before the
\end{abstract}

Electronic supplementary material The online version of this article (doi:10.1007/s00239-011-9469-9) contains supplementary material, which is available to authorized users.

T. J. P. van Dam · B. Snel

Theoretical Biology and Bioinformatics, Department of Biology,

Science Faculty, Utrecht University, Padualaan 8,

$3584 \mathrm{CH}$ Utrecht, The Netherlands

B. Snel

e-mail: B.Snel@uu.nl

T. J. P. van Dam · F. J. T. Zwartkruis · J. L. Bos Department Molecular Cancer Research, University Medical Center Utrecht, Universiteitsweg 100, 3584 CG Utrecht, The Netherlands

F. J. T. Zwartkruis

e-mail: G.J.T.Zwartkruis@umcutrecht.nl

J. L. Bos

e-mail: J.L.Bos@umcutrecht.nl

T. J. P. van Dam (ه)

Centre for Molecular and Biomolecular Informatics, Nijmegen

Centre for Molecular Life Sciences, Radboud University

Nijmegen Medical Centre, Geert Grooteplein 28,

6525 GA Nijmegen, The Netherlands

e-mail: Teunis.J.P.vanDam@gmail.com
Last Eukaryotic Common Ancestor and form a core to which new inputs have been added during animal evolution. In addition, we provide insight into how duplications and sub-functionalization of the S6K, RSK, SGK and PKB kinases shaped the complexity of the TOR pathway. In yeast we identify novel AGC kinases that are orthologous to the S6 kinase. These results demonstrate how a vital signaling pathway can be both highly conserved and flexible in eukaryotes.

Keywords TOR · Pathway evolution - Rheb · TSC1-2 complex $\cdot$ AGC kinases

\section{Introduction}

The target of rapamycin (TOR) is a major regulator of growth in eukaryotes (Thomas and Hall 1997). It integrates both intracellular signals that depend on nutrient availability, and extracellular signals, such as growth factors (Goberdhan et al. 2009; Oldham and Hafen 2003; Wullschleger et al. 2006). Therefore, dysfunction of TOR or other proteins in the TOR pathway is involved in organismal and cancer development in mammals (Menon and Manning 2008). The TOR protein is a kinase that participates in two distinct protein complexes. The TOR Complex 1 (TORC1) promotes translation by phosphorylating the S6 subunit of the ribosomal complex via ribosomal S6 kinase (S6K) and by phosphorylating the eukaryotic translation initiation factor $4 \mathrm{E}$ binding protein (4E-BP), which then dissociates from eIF4E allowing translation (Mahfouz et al. 2006; Tee and Blenis 2005). TORC1 is activated by the Ras homolog enriched in brain (Rheb) G-protein, which in turn is regulated by the tuberous sclerosis complex (TSC1/2 complex) (Inoki et al. 2003; 
Zhang et al. 2003). The best characterized activation route of TORC1 in animals is the insulin signaling pathway, which includes well characterized oncogenes and tumor suppressors such as phosphoinositide-3-kinase (PI3K), phosphoinositide-dependent kinase 1 (PDK1), phosphatase and tensin homolog (PTEN) and protein kinase B (PKB) (also known as AKT) (Engelman et al. 2006; Ma and Blenis 2009; Menon and Manning 2008; Shaw and Cantley 2006). It is not fully understood how TORC1 is regulated by nutrient levels (Avruch et al. 2009), although it is known that Rag GTPases play an important role in amino acid regulated TORC1 activity (Kim et al. 2008; Sancak et al. 2008). In addition S6K lowers PKB activity (Harrington et al. 2004; Kockel et al. 2010), creating a negative feedback by which TORC1 inhibits its own activation.

The TOR Complex 2 (TORC2) regulates cytoskeleton rearrangement in response to growth (Jacinto et al. 2004; Loewith et al. 2002). The regulation of TORC2 is mostly independent from TORC1 but involves PI3K signaling, activation by the ribosome (Zinzalla et al. 2011) and Rab GTPases (Tatebe and Shiozaki 2010). TORC2 positively regulates TORC1 via PKB (Cybulski and Hall 2009).

TOR is a conserved kinase and has been functionally characterized in animals (Hall 2008), fungi (yeast) (Lorberg and Hall 2004) and plants (Deprost et al. 2007). However, not all of the TOR complex subunits or TOR pathway components are equally conserved. For instance, the yeast Saccharomyces cerevisiae has retained the Rheb G-protein (Urano 2000), but lacks the Rheb regulatory genes Tuberous sclerosis 1 (TSC1) and Tuberous sclerosis 2 (TSC2), whereas Schizosaccharomyces pombe has retained all three genes (Matsumoto et al. 2002). Plants appear to lack Rheb and the TSC1/2 complex altogether (Díaz-Troya et al. 2008; Vernoud et al. 2003) despite the presence of TORC1 (Deprost et al. 2007; Menand et al. 2002).

To obtain novel insights into the evolution of the TOR pathway we performed phylogenetic analyses on the components of the TOR pathway, starting from the mammalian TOR pathway. We are specifically interested in the distribution of each subunit over all eukaryotes and the point of invention of single genes or even modules in evolution. To that end we searched in 64 diverse eukaryotic genomes for homologous and orthologous genes for components of the TOR pathway. The recent publication of genomes of highly divergent eukaryotic organisms such as Naegleria gruberi (Fritz-Laylin et al. 2010) now permits us to make detailed phylogenetic analyses of genes that play a key role in the TOR pathway and determine the earliest possible point of invention in evolution for each subunit. For a full overview of the species included in our analysis and how they are related to each other see Figure S4. We analyzed each evolutionary or phylogenetic profile in light of distinct components of the pathway (e.g., the TOR complexes, Rheb regulation) and the pathway as a whole.

Importantly, we found that TOR, all subunits of TORC1 and 2 and a large part of the TOR pathway components form an evolutionary core. New regulatory inputs, such as insulin and TNF $\alpha$ signaling, have been added to the core TOR pathway during animal evolution. We show that TORC1 and TORC2 appear to behave as independent evolutionary modules, even though the majority of the subunits are shared between the two complexes. We infer the presence of a large common evolutionary core, including Rheb and TSC2, in the Last Eukaryotic Common Ancestor (LECA), the last ancestral eukaryote that gave rise to all current eukaryotic species. We provide an indepth phylogenetic analysis of TOR, Rheb, TSC2 and translationally controlled tumor protein (TCTP) as well as other components of the TOR signaling pathway. In addition, we reveal the remarkable role of the duplications of an ancestral AGC kinase that gave rise to the $\mathrm{S} 6 \mathrm{~K}$, ribosomal protein S6 kinase (RSK), PKB and serum/glucocorticoid regulated kinase (SGK) kinases, in the increasing complexity of the TOR pathway. We conclude that a vital signaling pathway can be both highly conserved and flexible in eukaryotes.

\section{Results and Discussion}

The Evolution of TOR Complex 1 and 2 are Decoupled

TOR functions as part of two distinct protein complexes: the TORC 1 and TORC 2 complexes (Loewith et al. 2002). In mammalian cells TORC1 contains mTOR, Lethal with Sec Thirteen (LST8) and Raptor, while TORC2 contains mTOR, LST8, Raptor independent companion of TOR (Rictor) and stress-activated MAP kinase interacting protein (SIN1) (Fig. 1). TOR and LST8 are both present in genomes in all major eukaryotic lineages and therefore form the evolutionary core of the TOR complexes (Fig. 1). In addition, we also observe that TOR and LST8 co-occur with either Raptor (TORC1) or Rictor (TORC2) or both, indicating that both TOR complexes are old and were likely present in LECA, the common ancestor of all current eukaryotic species.

We find TORC1 together with TORC2 in all major lineages, except plants, which possess only TORC1 (Fig. 1). Interestingly we detect TORC2, but not TORC1 in the ciliates Tetrahymena thermophila and Paramecium tetraurelia. It therefore appears that the two distinct TOR complexes are decoupled in evolution as either one can be lost while the other is maintained.

We do not detect any of the TOR complex subunits in the microsporidium Encephalitozoon cuniculi and the 


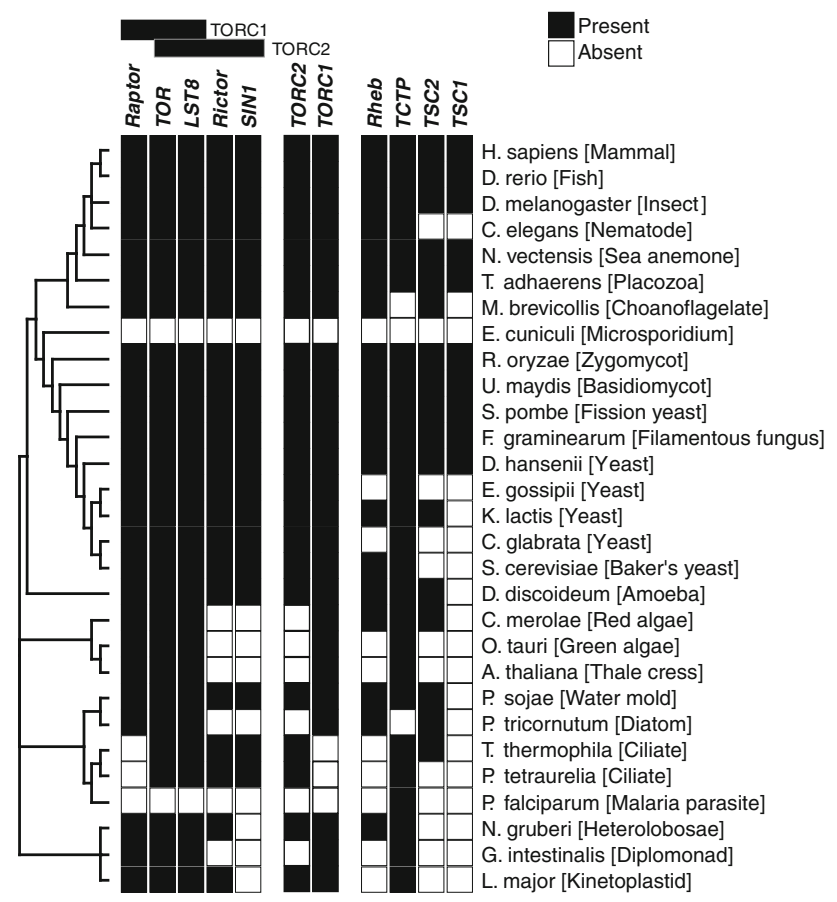

Fig. 1 Absence/presence plots in a subset of 65 eukaryotic genomes. Animals and fungi have both TORC1 and TORC2 while plants have only TORC 1 and ciliates have only TORC2. Apparently it is possible to lose either one of the complexes while maintaining the other. The GAP domain of TSC2 is well conserved and is found (with few exceptions) in species that also contain Rheb throughout the eukaryotic lineages. TSC1 is an animal/fungal invention and therefore newer than TSC2 and Rheb. The occurrence of TCTP in species lacking Rheb and vice versa, raises additional doubt on the debated Guanine Exchange Factor function of TCTP

apicomplexa Plasmodium falciparum, Cryptosporidium parvum and Theileria parva indicating at least two independent loss events for TOR signaling in eukaryotes. All four species are intracellular parasites and have reduced genomes and cellular structure. Host-parasite interactions might have replaced the function of TOR in these organisms as growth of the parasite is directly linked to conditions in the host cell.

Distinct protein complexes that share subunits (i.e., hyperlinks) can provide a selective reason for maintaining duplicate copies of these shared subunits (Shevchenko et al. 2008). Therefore, we could expect to find duplications of TOR in some species, resulting in a dedicated TOR for each of the two TOR complexes. Indeed we find duplications of TOR in Saccharomyces cerevisiae, Candida glabrata, Schizosaccharomyces pombe, Batrachochytrium dendrobatidis, Populus trichocarpa, Emiliania huxleyi, Trypanosoma brucei, Leishmania major, Phytophthora infestans and Phytophthora sojae (see Figure S1). The duplication of TOR in $S$. cerevisiae and C. glabrata likely originates from the Whole Genome Duplication event. In S. cerevisiae both TOR1 and TOR2 can be part of TORC1, while TOR2 is specific for the TORC2 (Loewith et al. 2002), which indicates that the two TOR duplicates are not completely functionally diverged. The TOR duplication in S. pombe and B. dendrobatidis are lineage-specific duplications and occurred independently from each other and from the duplications in S. cerevisiae and $C$. glabrata. While we have no functional descriptions for $B$. dendrobatidis, it has been shown for $S$. pombe, that TOR1 and TOR2 function as part of TORC1 (Hartmuth and Petersen 2009), while TOR1 is specific for TORC2 (Otsubo and Yamamato 2008). Note, that the naming of TOR1 and TOR2 in S. cerevisiae and S. pombe can cause confusion as the genes resulted from independent duplication events, the naming of TOR 1 and TOR 2 in both yeasts does not reflect one-to-one orthologous relationships but is based on order of discovery (Soulard et al. 2009). Surprisingly, LST8 has not been duplicated in any of the species examined. This raises an interesting question: why do the two hyperlinks TOR and LST8 behave differently in evolution? We hypothesize that duplication and subsequent functional divergence of LST8 may have implications for the structural integrity of the two TOR complexes, while minor modifications to the TOR duplicate genes increased functional divergence without compromising complex stability.

TSC2-Rheb Signaling, a Highly Conserved Signaling Route to TORC1

\section{The Rheb G-protein, Conserved Throughout the Eukaryotic} Lineage

The Rheb G-protein is one of the major regulators of TOR activity in animals and directly regulates the activity of TORC1 but not TORC2 (Cybulski and Hall 2009). Rheb is a Ras-like small GTPase and the sequences of small GTPases are highly conserved (Colicelli 2004; Wennerberg et al. 2005). We previously reconstructed the phylogeny of the Ras-like small GTPases (van Dam et al. 2011). From this phylogeny we identified Rheb orthologs (see "Methods" section; Figure S2) and derived a phylogenetic profile of orthologs (Fig. 1). We identified Rheb orthologs in all animals and fungi (except in C. glabrata, Eremothecium gossypii and E. cuniculi). Additionally we identify ortho$\operatorname{logs}$ in distantly related organisms such as diatoms, oomycetes, the amoeba Dictyostelium discoideum, the heterolobosida Naegleria gruberi and the red algae Cyanidioschyzon merolae. This is the first time, to our knowledge, that a G-protein belonging to the Ras-like subfamily of small GTPases has been identified in the Archaeplastida (i.e., plants and red and green algae).

The identification of Rheb orthologs in distantly related species strongly suggests that Rheb originated in or before 
LECA. We observe Rheb orthologs in species that also contain TORC1, which indicates that the regulation of TOR by Rheb is strongly conserved. However, we do not observe the opposite, e.g., species that have TORC1 do not necessarily have a Rheb ortholog. The most notable of these species are the green algae and plants, but also the yeasts $C$. glabrata and E. gossypii. The presence of a Rheb ortholog in $C$. merolae indicates a loss of Rheb in the ancestor of the green algae and plants.

\section{TSC1 is an Animal-Fungal Innovation} in TSC2 Regulation of Rheb

Next we investigated the phylogeny of the only known regulator of Rheb, the GTPase activating protein (GAP) TSC2 (data on TSC2 is also presented in (van Dam et al. 2011) in light of Ras signaling and other RapGAP domain containing proteins). TSC2 integrates many inputs such as MAPK/Ras signaling via RSK1 (Ma et al. 2005; Roux et al. 2004), Wnt signaling via GSK3 $\beta$ (Inoki et al. 2006) and insulin signaling via PI3K and PKB (Zoncu et al. 2011) in animals. The TSC2 GAP domain occurs in all major eukaryotic super groups, except the excavates, indicating it much older than previously suggested (Serfontein et al. 2010) and likely originated in or before the LECA. We find the TSC2 GAP domain orthologs in species that also have a Rheb ortholog, including the red algae $C$. merolae mentioned above. The only exception is the ciliate $T$. thermophila. Therefore, we predict that the TSC2 GAP orthologs will regulate the Rheb orthologs in D. discoideum, C. merolae and the Phytophthora species.

Furthermore, we find that while TSC1 orthologs are always observed together with TSC2 orthologs in the same genomes, TSC2 can be found on its own in additional eukaryotic species (Fig. 1). Interestingly in some of these species (D. discoideum, C. merolae, P. infestans, $P$. sojae, Phaeodactylum tricornutum) we were able to identify the GAP domain but not the Tuberin domain that is necessary to dimerize with TSC1. Strikingly, we find TSC1 orthologs in animals and fungi, which is the same phylogenetic distribution as the tuberin domain of TSC2. Therefore, it is likely that TSC1 itself and the ability of TSC2 to dimerize with TSC1 via the tuberin domain are inventions in the Opisthokont ancestor (i.e., in the animal and fungal ancestor).

The absence of the TSC $1 / 2$ complex in C. elegans and S. cerevisiae (Fig. 1) suggests that caution should be taken when comparing regulatory mechanisms of TOR between these two and other species. For instance, regulatory mechanisms for Rheb and TOR discovered in animals, such as in D. melanogaster do not necessarily hold for C. elegans and vice versa.
Translationally Controlled Tumor Protein 1, No Phylogenetic Linkage with Rheb

TCTP (also known as TPT1) has been reported to be the Guanine Exchange Factor (GEF) for Rheb (Hsu et al. 2007) but this function has been debated by us and others (Rehmann et al. 2008; Wang et al. 2008). Hence it is interesting to study the phylogenetic profiles of TCTP and TSC2 and compare them to Rheb (Fig. 1). We constructed the phylogenetic profile of TCTP and found that TCTP is present in nearly all of the eukaryotic genomes we studied including plants, chromalveolata and excavata. Interestingly we observe eukaryotic species which have a TCTP ortholog but do not have the Rheb G-protein (i.e., green plants and algae, apicomplexa, ciliates, C. glabrata, E. gossypii, see Fig. 1) and vice versa (i.e., the choanoflagellate $M$. brevicollis and the diatom $P$. tricornutum). In addition we observe in the apicomplexa (i.e., a group of unicellular intracellular parasites including Plasmodium falciparum), that even though they contain a TCTP ortholog, they do not only lack Rheb but also TOR.

In a previous study on Ras Guanine Exchange Factors (RasGEFs) we observed a strong evolutionary link between the RasGEFs and their respective Ras-like GTPases (van Dam et al. 2009). The RasGEF functional domain, the CDC25 homology domain, and the Ras, Rap and Ral GTPases were found to be present or absent together in a diverse set of eukaryotic genomes. In contrast we observe no such strong presence/absence pattern for TCTP and Rheb. It therefore seems that there is no evolutionary linkage between TCTP and Rheb.

Further doubts about TCTP's GEF activity for Rheb arise from experimental evidence of TCTP function in Arabidopsis thaliana when put in an evolutionary framework. Berkowitz et al. (2008) studied the function of the ortholog of TCTP in A. thaliana. They found that TCTP acts as an important regulator of growth, and implied that TCTP functions in TOR activity, which resembles the situation in animals (Hsu et al. 2007). The similarity between plant TCTP and animal TCTP is interesting because Arabidopsis does not have a Rheb ortholog (Vernoud et al. 2003 and this study). Berkowitz et al. postulate that Arabidopsis TCTP regulates another GTPase (either a Rhop- or Rab-like G-protein) which might function in an equivalent way to Rheb. We are of the opinion that the results of Berkowitz et al., that TCTP functions as a positive regulator on TOR activity in A. thaliana in the absence of Rheb, indicate that TCTP might in fact not be a RhebGEF, but instead suggest that TCTP regulates TOR via an alternative route. 


\section{Evolution of the Mammalian TOR Pathway: Gaining Inputs}

We extended our phylogenetic study to include upstream and downstream components of the mammalian TOR pathway and thereby put Rheb and TORC in a wider biological context. We focus on the mammalian TOR pathway because TOR signaling in animals and particularly mammals is the most extensively studied intact TOR pathway (e.g., not lacking key components such as the TSC1/2 complex in S. cerevisiae or TORC2 in A. thaliana). The TOR pathway was assembled from literature to reflect current consensus. For each protein we constructed the phylogenetic profile and determined the point of invention (e.g., the age of a protein. See Table S2 for all phylogenetic profiles). We depict the age of each protein along the metazoan natural history towards LECA in the representation of the TOR pathway (Fig. 2).

Recently, Serfontein et al. (2010) published an evolutionary survey on the components of the TOR pathway in a representative selection of eukaryotic genomes. Our results concerning the evolution of the TSC1/TSC2-TOR pathway underline some observations made by Serfontein and coworkers but differ considerably in others. While Serfontein and coworkers find that the evolutionary "core" of the pathway that was present in LECA consisted out of the TORC1 complex (TOR, LST8, Raptor), AMP-activated protein kinase (AMPK), PI3K and PTEN and S6K, we find that TORC2 (TOR, LST8, Rictor, SIN1), Rheb, TSC2, PDK1 and the remaining AGC kinases PKB, RSK and SGK are also part of this evolutionary core. We show that Rheb and TSC2 have not been "bolted on" in evolution but are in fact part of the evolutionary core that originated in or before LECA. We do, however, observe other regulatory elements of the TOR pathway that have been added at a later evolutionary stage. We made two observations concerning the evolution of new TOR signaling regulation.

The first observation is that the regulation of TOR activity by insulin is an animal-specific addition to the pathway. The second observation is that the more recently invented TSC1 introduces novel regulatory input onto the Rheb-TOR cascade (i.e., FIP200 and IKK $\beta / T N F \alpha$ ). These two observations suggest that although TOR signaling is highly conserved within eukaryotes, it is also flexible enough to accept new inputs and can be adapted to suit new environments (e.g., multicellular tissues).

The animal-specific addition of insulin signaling to the TOR pathway could be an adaptation to account for cellular growth in a multicellular environment. The growth and divisions of individual cells need to be regulated system wide and insulin might very well play the role of system-wide growth control via TOR. Stimulation by insulin has been a fruitful method to investigate TOR

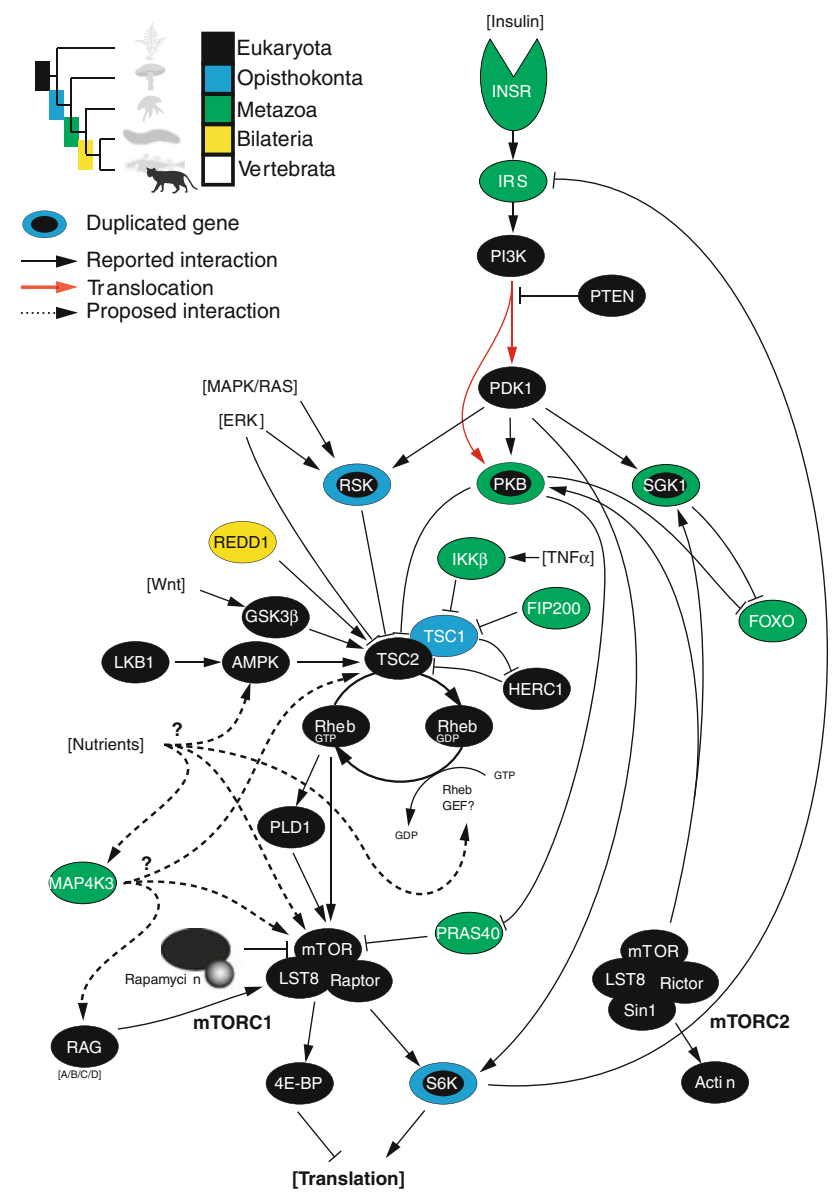

Fig. 2 Evolution of the mTOR pathway. We reconstructed the mTOR pathway based on literature. The indicated age of each gene is based on a reconstruction from phylogenetic profiles. For duplicate genes the outer circle denotes the time of duplication and the inner circle denotes the origin of the complete orthologous group. The regulation of TOR via insulin and TNF $\alpha$ are animal-specific additions onto an ancient TOR pathway. The invention of TSC1 in the animal and fungal ancestor allowed for new regulatory inputs onto TSC2. Duplications of an ancestral AGC kinase that gave rise to S6K, RSK, PKB and SGK played a significant role in the evolution of the TOR pathway

signaling in animal models, but as a model of TOR activation and regulation it is distinct from our knowledge of TOR in non-animal model species. It would be interesting to investigate if or in what way TOR is regulated by system-wide growth control (other than nutrient availability) in plants, a group of organisms that have separately acquired multicellularity.

Ma et al. (Ma and Blenis 2009) suggested that PRAS40 might represent a conserved $\mathrm{PKB}$ regulation route to TORC1 while TSC1/2 represented a newer additional pathway in higher eukaryotes because TSC1 and TSC2 were previously not found in lower eukaryotes like $S$. cerevisiae. However, due to the availability of many newly sequenced eukaryotic genomes and with more extensive phylogenetic profiling as done here, it becomes evident that 
PRAS40 represents an animal-specific additional route of PKB-TORC1 activation and that the Rheb/TSC1/TSC2 route is in fact much older.

Serfontein and coworkers (Serfontein et al. 2010) have detected PKB orthologs only in animals, amoebozoa and excavate species, but not in plants and chromalveolates. However, they have included only those sequences that included both the kinase domain and a Pleckstrin Homology (PH) domain that is characteristic of the animal PKB. However, the PH domain is known to be very promiscuous in eukaryotes (Basu et al. 2008) and is therefore not suitable to use as a restriction criterion for orthology.

We find that the AGC kinases PKB and SGK are paralogs that have arisen from a duplication event in the animal and choanoflagellar ancestor, but we also find PKB/SGK orthologs in chromalveolates and excavates. PKB and SGK share common ancestry with the AGC kinases S6K and RSK, which are also involved in TOR signaling. This suggests that the AGC kinases and duplication events play a striking and complex evolutionary role in the TOR pathway. We therefore focused on the evolution of the AGC kinases.

\section{Duplication of AGC Kinases has Increased Internal TOR Pathway Complexity}

In the mTOR pathway, the AGC family kinases S6K, RSK1, PKB and SGK1 are located both upstream and downstream of TOR. S6K and RSK1 arose from a duplication event in the ancestor of animals and fungi (Opisthokont ancestor) while PKB and SGK1 arose from a duplication event in the ancestor of animals and the closely related choanoflagellate Monosiga brevicollis (ShalchianTabrizi et al. 2008) (see Fig. 3a; Figure S3). The S6K-RSK and PKB-SGK ancestral genes themselves have arisen from a gene duplication in or before LECA. The evolutionary relation between $\mathrm{S} 6 \mathrm{~K}, \mathrm{RSK} 1, \mathrm{PKB}$ and SGK1 make it uniquely possible to reconstruct the evolution of their regulatory interactions within the TOR pathway. In Fig. 3 we have reconstructed the TOR pathway at several points in evolution based on events in the evolution of the AGC kinases and experimental characterization of orthologous genes in $H$. sapiens, $S$. cerevisiae, $S$. pombe and A. thaliana.

\section{Duplication of the PKB-SGK1 Ancestral Gene \\ in the Ancestor of Animals and Choanoflagellates}

The PKB and SGK genes duplicated from a single ancestral gene in the filozoan ancestor, i.e., the ancestor of animals and choanoflagellates (see Fig. 3a; Figure S3). However, additional new components have been invented specifically in animals, such as the insulin and $\mathrm{TNF} \alpha$ signaling pathways. Therefore, we reconstructed the TOR pathway in the metazoan ancestor. PKB and SGK are both activated by PDK1 (Alessi et al. 1997; Burgering and Coffer 1995; Kobayashi and Cohen 1999; Park et al. 1999) and the TORC2 complex (Hresko and Mueckler 2005; Jones et al. 2009; Sarbassov et al. 2005; Soukas et al. 2009). In addition, PKB is activated by translocation to the plasma membrane upon PI3K activation (Stokoe 1997) (Fig. 3b). It is therefore very likely that the PKB/SGK ancestral protein was also activated by PDK1 and TORC2 ancestral proteins. PI3K-dependent translocation of PKB is likely a new function acquired by PKB.

PKB inhibits the TSC1/2 complex by phosphorylating TSC2 (Dan et al. 2002; Potter et al. 2002) and inhibits FOXO transcription factors by directly phosphorylating them (Burgering and Kops 2002). SGK also inhibits FOXO by phosphorylation (Brunet et al. 2001) but has not been reported to phosphorylate TSC2. We can partly derive the ancestral functions by comparing PKB and SGK functions to the gene functions of the co-orthologous genes in $S$. pombe and $S$. cerevisiae.

In $S$. pombe and $S$. cerevisiae there are three coorthologous genes to both PKB and SGK (Sck1, Sck2 and Gad8 in $S$. pombe, YPK1, YPK2 and SCH9 in S. cerevisiae, see Figure S3). The S. pombe and S. cerevisiae PKB/ SGK1 orthologs Sck1, Sck2, Gad8 and SCH9 are implied to have function in oxidative stress responses and aging (Chen and Runge 2009; Ikeda et al. 2008), similar to PKB and SGK1 in animals. Therefore, the role of PKB and SGK in regulating longevity is conserved and likely an ancestral function in the Opisthokont ancestor (Fig. 3c).

In contrast to stress response and aging, the origin of TSC2 phosphorylation by PKB is not immediately apparent. In $S$. cerevisiae TSC2 has been lost and we have been unable to find any references that implicate the $S$. pombe PKB/SGK orthologs Sck1, Sck2 in growth regulation via TOR (Sck1/2) or that Gad8 has been associated with the TSC1/2 complex. This makes it difficult to determine if TSC2 phosphorylation is an ancestral function or whether it has been specifically acquired by PKB. Nevertheless, there are similarities in function of $\mathrm{PKB}$ with RSK, and we can therefore reconstruct the ancestral function of PKB and SGK by comparing their functions to their paralogs RSK and S6K.

Similar to PKB, RSK also inhibits the TSC1/2 complex by phosphorylating TSC2 in mammals (Roux et al. 2004). Because the GAP domain of TSC2 is conserved throughout the eukaryotic lineage, the most plausible scenario is that PKB and RSK inhibition of TSC2 is an ancestral function of the PKB-SGK-S6K-RSK ancestral gene (henceforth we will refer to this ancestor as the ancestral AGC kinase for brevity). In this scenario the TSC2 regulation is an ancestral function maintained by PKB and lost by SGK (Fig. 3b).

Interestingly, similar to the filozoan PKB-SGK duplication event, fungi seem to have undergone a similar duplication event of the ancestral PKB-SGK kinase. YPK1 
A

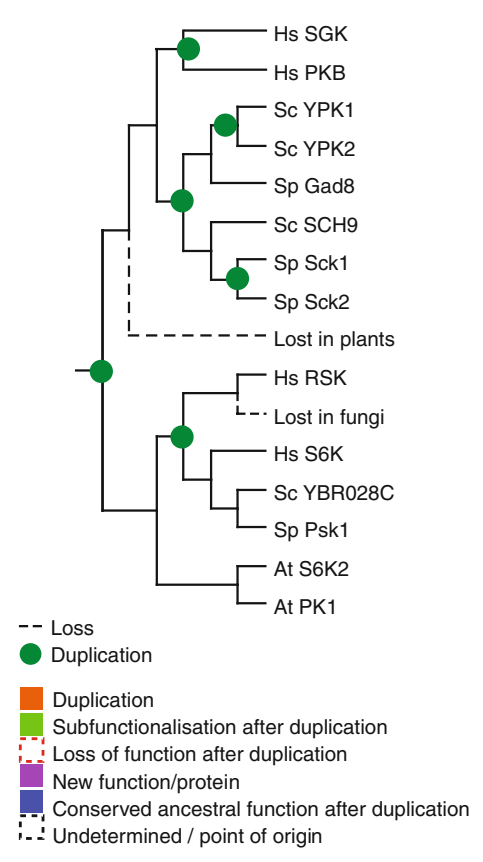

B

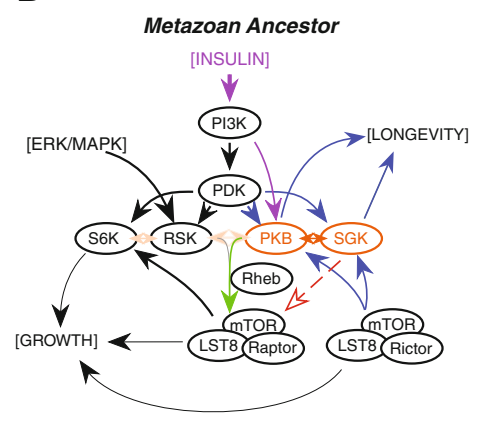

D

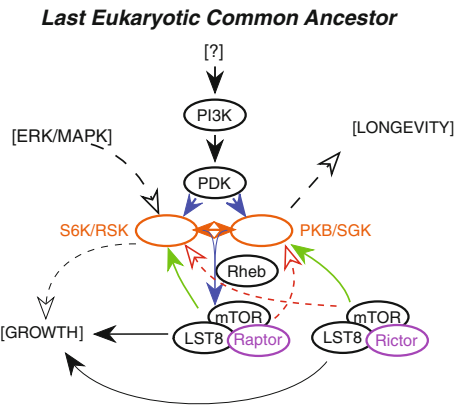

C

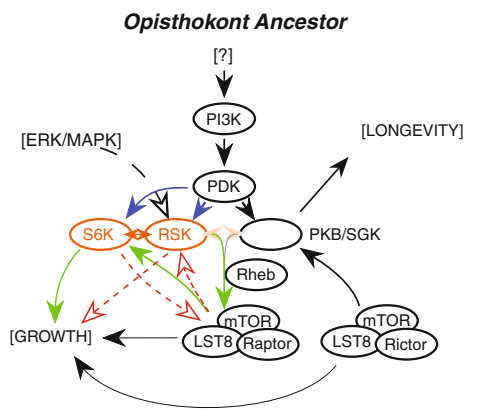

E

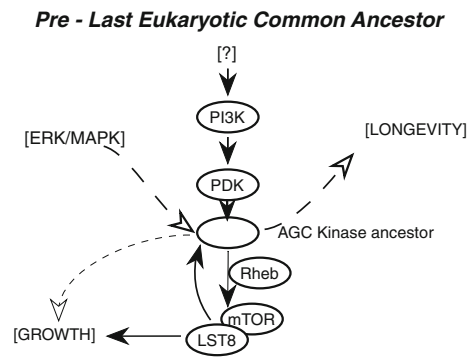

Fig. 3 Evolutionary reconstruction of the ancestral TOR signaling pathway based on the evolutionary reconstruction of the AGC kinase ancestral genes of S6K, RSK1, PKB and SGK1. a Simplified representation of the phylogenetic tree of the AGC kinases (see Figure S3). Species indication: Hs Homo sapiens, Sc S. cerevisiae, Sp $S$. pombe, At A. thaliana. b Reconstruction of TOR signaling in the animal ancestor. An AGC kinase duplicated in the ancestor of animals and choanoflagellates to give rise to PKB and SGK. SGK lost the ability to inhibit TSC2. New signaling inputs were invented in the animal ancestor, among which is the insulin signaling. c TOR signaling in the ancestor of animals and fungi. An AGC kinase duplicated in the ancestor of animals and fungi (Opisthokont

and YPK2 function in S. cerevisiae can be rescued by rat SGK, but not mouse PKB or rat S6K (Casamayor et al. 1999). Therefore, although the PKB-SGK and YPK-SCH9 duplication events in animals and fungi occurred independently, the resulting animal and fungal paralogs appear to have evolved in a functionally similar way.

\section{Duplication of the S6K-RSK1 Ancestral Gene in the Animal and Fungal Ancestor}

The S6K and RSK genes duplicated from a single ancestral gene in the fungal and animal ancestor (Fig. 3c). Like PKB and SGK, both are regulated by PDK1 (Jensen 1999; Pullen et al. 1998). However, S6K and RSK are not regulated by the TORC2 complex (Jacinto and Lorberg 2008). S6K is regulated by the TORC1 complex instead (Burnett et al. 1998), while RSK is regulated via MAPK signaling (Carrière et al. 2008). While it is very likely that the S6KRSK ancestral protein was regulated by the PDK1 ancestral protein, the regulation of S6K by TORC1 and RSK by ancestor) that give rise to S6K and RSK. The duplication was followed by subsequent sub-functionalization of ancestral functions between the two paralogs. d TOR signaling in LECA. The duplication of the ancestral AGC kinase that give rise to the RSK-S6K and PKBSGK ancestral precursor genes corresponds to the differential activation by either TORC1 or TORC2, respectively. e Reconstruction of pre-LECA TOR signaling. The shared subunits TOR and LST8 of both TOR complexes suggest that before LECA there was at one point only one proto-TOR complex. As the AGC kinases also share single ancestry we can reduce the complexity of the TOR pathway in pre-LECA even further

MAPK initially obscures whether the ancestral kinase was either activated by TORC1 or MAPK or both.

We can infer whether the ancestral protein was activated by TORC1 and/or MAPK by inferring the ancestral function from experimental evidence for TOR signaling in the plant $A$. thaliana. RSK1 and S6K are co-orthologs to the A. thaliana S6K kinase. The S6K kinase of A. thaliana is regulated by the Arabidopsis TOR complex (Mahfouz et al. 2006), which has the same composition as TORC1 in other organisms, and we can therefore infer that the S6K-RSK ancestral protein was likely activated by TORC1. Thus S6K maintained the ancestral regulation by TORC1, i.e., RSK has lost the regulation by TORC 1 (the dashed edge in Fig. $3 \mathrm{c}$ from TORC1 to RSK). Activation by MAPK of the ancestral protein cannot be inferred from the Arabidopsis S6K as there is no published link between Arabidopsis MAPK and Arabidopsis TOR signaling but we cannot exclude loss of this function in plants (the dashed edge from MAPK in Fig. 3c). Previously we deduced that RSK regulation of TORC1 activity via TSC 2 is likely an ancestral function from the symmetry with PKB. 
Therefore, TSC2 regulation by RSK is an ancestral function that has been lost in S6K (the dashed edge in Fig. 3c from S6K to TORC1).

Intriguingly, both S. pombe and S. cerevisiae have a oneto-one ortholog to S6K that has not been fully characterized yet, or only superficially (see Fig. 3a; Figure S3). The $S$. cerevisiae locus YBR028C codes for a kinase, but has not been reported in the literature. The S6K ortholog in S. pombe, $p s k 1$, has been reported to be involved in phenylarsine oxide resistance and disruption of the $p s k 1$ gene did not result in growth defects (Mukai 1995). Instead, the S6K-like cellular function in S. cerevisiae has been ascribed to SCH9 (Urban et al. 2007), which according to our analysis is an ortholog of mammalian PKB and SGK. SCH9 shows that the AGC kinases are capable of performing cellular functions that have been ascribed to their paralogs, possibly increasing the complexity of the roles the AGC kinases play in TOR signaling. Given that the poorly characterized YBR028C gene in S. cerevisiae is clearly orthologous to $\mathrm{S} 6 \mathrm{~K}$, we suggest that there might be a substantial role for this gene to be uncovered in TOR signaling.

\section{Back to the Root: The ancestral AGC Kinase and the Ancestral TOR Pathway}

Above we have described the ancestral states of the S6KRSK and PKB-SGK ancestral genes. We observe symmetric functions between the two ancestral genes and therefore we are able to (partly) reconstruct the functions of the ancestral AGC kinase (e.g., the ancestral gene of S6K, RSK, PKB and SGK). All four kinases are regulated by PDK1 and we can therefore infer that the ancestral AGC kinase was also regulated by the PDK1 ancestral gene (Fig. 3d). Above, we also deduced that the S6K-RSK and PKB-SGK ancestral genes possibly regulated TORC1 activity via the TSC2 ancestral gene as it is a shared function of both RSK and PKB (Fig. 3d).

Phosphorylation of the S6 ribosomal subunit by the S6K-RSK ancestral kinase cannot be reconstructed beyond the LECA, because the PKB and SGK kinases do not share this function and we are therefore unable to determine if the S6 activation was a function of the ancestral AGC kinase that has been lost by the PKB-SGK ancestral gene or that it is an acquired function of the S6K-RSK ancestral gene (the dashed edge from S6K/RSK in Fig. 3d).

Our phylogenetic reconstruction suggests the existence of a TORC1 and a TORC2 complex in LECA that functions in conjunction with Rheb/TSC2 to activate at least two distinct AGC kinases (Fig. 3d). These AGC kinases arose from duplication and thus required (partially) independent regulation by TOR for their sub-functionalization (compare Fig. 3e and d). TORC1 and TORC2-specific proteins like Raptor and Rictor most likely contributed situation-specific activation of AGC kinases, i.e., determine context-relevant downstream outputs.

The positive feedback loop that emerges in the reconstruction of the ancestral TOR pathway in LECA (Fig. 3d, e) imposes a network structure that is undesirable as the feedback loop could easily result in a constitutively activated TOR and therefore uncontrolled growth. In animals negative feedback from S6K and TOR to PKB or more upstream elements in the insulin pathway have been documented (Findlay et al. 2005; Kockel et al. 2010; Manning 2004). It is very likely, given the importance of proper regulation of the TOR pathway that negative feedback routes were also in place in LECA. However, we are unable to reconstruct these negative feedbacks back to LECA. An explanation for this could be that the negative feedbacks in the TOR pathway have been subject to change in evolution. Subsequent duplications of the ancestral AGC kinase and sub-functionalization, however, might have provided the opportunity to increase the possibility for more precise or additional regulation of TOR activity.

\section{Flexibility in a Conserved Signaling Pathway}

The TOR pathway is a universal regulator of cell growth in eukaryote species. TOR is the master regulator and integrates many inputs such as growth signals and nutrient availability in a cell. We show that the two TOR complexes are highly conserved and were already present in the last common ancestor of all eukaryotes. We analyzed the origin and evolution of each subunit of the TOR complexes separately as well as other components of the TOR signaling pathway. We show that TORC 1 and TORC2 behave as separate evolutionary modules that can be individually lost [i.e., loss of either Raptor (TORC1) or Rictor (TORC2) or both complexes as a whole]. We find that the TOR pathway has a conserved "core" to which new inputs have been added early in animal evolution, such as insulin and $\mathrm{TNF} \alpha$ signaling. We also find evidence that the core itself has been extensively modified in evolution by duplications of ancestral AGC kinases that gave rise to S6K, RSK, SGK and PKB. The evolution of TOR and conversely the whole TOR pathway demonstrates that a vital signaling pathway can be both highly conserved and flexible in eukaryotes and can be adapted to fulfill changing requirements of growth regulation by eukaryotic organisms.

\section{Methods}

Genome Selection

We acquired protein sequences (proteomes) of 64 divergent eukaryotic species from EnsEMBL (Hubbard et al. 
2009), JGI, the Broad institute or their respective genome project sites. Proteomes filtered for "best model" were downloaded when available and longest sequences were selected in case of multiple transcripts per gene. We have selected a wide range of animal and fungal genomes as most research on TOR signaling is being done in either animal or fungal model organisms. We also included a wide range of genomes belonging to other major super groups, such as the Archeaplastida, Chromalveolates and Excavates, to be able to accurately time the origin of each TOR pathway component. For a full overview of genomes, source and version information see Table S1. For a species tree of the used genomes, see Figure S4.

\section{Phylogenetic Analyses}

\section{Orthology Determination and Phylogenetic Profiles}

Orthology was determined automatically by applying MCL (Van Dongen 2008) on InParanoid (Remm et al. 2001) species-species comparisons. We performed an all versus all BLAST (Altschul et al. 1990) on the whole set of 64 genomes with the options -p blastp -m 8 -v $1000000-b$ 1000000. The BLAST results were then split into separate data files as required for InParanoid. InParanoid was applied to all possible combinations of the 64 genomes using the default settings (score_cutoff $=50$, outgroup_cutoff $=50$, seq_overlap_cutoff $=0.5$, conf_cutoff $=0.05$, group_overlap_cutoff $=0.5$, grey_zone $=0$ ). A matrix dataset for MCL was constructed from the InParanoid analyses by constructing edges between genes for each InParanoid cluster taking the lowest confidence value of both genes. Edges were only drawn between genes of different species. The MCL analysis was run with parameters -abc -I 1.5 -write-graph.

Phylogenetic profiles were constructed from the InParanoid-MCL clusters by determining which species were (not) represented in each cluster. The phylogenetic profiles of genes of interest were manually verified.

\section{Rheb Orthology Identification and Phylogenetic Analysis}

The sequences of the selected genomes were searched using the Pfam (Finn et al. 2008) HMM profile for the Ras family (Pfam accession PF00071.12, Pfam version 23) and hmmsearch of the HMMER package (Eddy 1998) version 2.3.2. All sequences with a bitscore larger than 0 were selected. Due to the high sequence similarity of Ras to other small GTPases many other small GTPases are included in this set. An alignment of all sequences was made using the MAFFT program (Katoh et al. 2002) with the -globalpair option. A neighbor joining tree was constructed using the Quicktree program (Howe et al. 2002). A sub-tree was selected which contained all Ras-like subfamily members but no other small GTPase. The sequences were gathered from the initial alignment as manual inspection of the alignment produced from the subset showed it was suboptimal to the initial alignment. Subsequently a phylogenetic tree was constructed over all Raslike subfamily members using RAxML (Stamatakis et al. 2005) (-T 4 -x 488761235 -f $a-N 1000$-m PROTGAM$M A I W A G)$. From the resulting tree a sub-tree was selected that contained Rheb and as many genes from the searched species as possible without including other known small GTPases. All phylogenetic trees were visualized using Dendroscope (Huson et al. 2007). The data on Rheb phylogeny has also been presented in (van Dam et al. 2011).

\section{The AGC Kinases SGK1, PKB, RSK1 and S6K}

Protein sequences belonging to the cluster of orthologous groups that contains S6K, RSK, PKB and SGK, were aligned using MAFFT (Katoh et al. 2002) with the -globalpair option. The resulting alignment was analyzed and a segment that showed high sequence similarity between all sequences was used for further analysis (positions 893-2050, corresponding to the kinase domain and $\mathrm{PKC}$ terminal domain). A phylogenetic tree, including bootstrap analysis, was constructed using RAxML (Stamatakis et al. 2005) (-T 8 -x 78382369 -f a -N 1000 -m PROTGAMMAIWAG).

\section{TSC2 Ortholog Identification}

TSC2 orthologs were initially identified using the automated orthology determination as described above. Closer examination revealed that the sequences only had the GAP domain sequence in common. Since the GAP domain of TSC2 belongs to a larger family of GAP domains, the Ran/ RapGAP domain family, we used phylogenetic methods to faithfully determine true orthology based on the RapGAP domain sequences. We gathered RapGAP domain sequences from the sequence set by using a custom-made HMM model and hmmsearch of the HMMER package (Eddy 1998) version 2.3.2. The domain sequences were aligned using MAFFT (Katoh et al. 2002) -globalpair. A phylogenetic tree was built using RAxML (Stamatakis et al. 2005) (-T 6 -x 23421421 -f a -N 1000 -m PROTGAMMAIWAG). The data on TSC2 phylogeny has also been presented in (van Dam et al. 2011).

Acknowledgments We want to thank Prof. Michael Hall and Thomas Sturgill for their suggestions and critical appraisal, Jos Boekhorst, Gabino Sanchez-Perez, Like Fokkens and Michael Seidl for their help in performing the analyses and support. The sequence data of selected genomes were produced by the US Department of Energy Joint Genome Institute http://www.jgi.doe.gov/, in collaboration with 
the user community, or the Fungal Genome Initiative of the Broad Institute. For a full overview of the genomes and references see Table S1. This work was supported by the BioRange program of the Netherlands Bioinformatics Centre (NBIC), which is supported by a BSIK grant through the Netherlands Genomics Initiative (NGI).

Open Access This article is distributed under the terms of the Creative Commons Attribution Noncommercial License which permits any noncommercial use, distribution, and reproduction in any medium, provided the original author(s) and source are credited.

\section{References}

Alessi DR, James SR, Downes CP et al (1997) Characterization of a 3-phosphoinositide-dependent protein kinase which phosphorylates and activates protein kinase $\mathrm{B} \alpha$. Curr Biol 7:261-269. doi: 10.1016/S0960-9822(06)00122-9

Altschul SF, Gish W, Miller W et al (1990) Basic local alignment search tool. J Mol Biol 215:403-410. doi:10.1006/jmbi.1990. 9999

Avruch J, Long X, Ortiz-Vega S et al (2009) Amino acid regulation of TOR complex 1. Am J Physiol Endocrinol Metab 296:E592E602. doi:10.1152/ajpendo.90645.2008

Basu MK, Carmel L, Rogozin IB, Koonin EV (2008) Evolution of protein domain promiscuity in eukaryotes. Genome Res 18:449-461. doi:10.1101/gr.6943508

Berkowitz O, Jost R, Pollmann S, Masle J (2008) Characterization of TCTP, the translationally controlled tumor protein, from Arabidopsis thaliana. Plant Cell 20:3430-3447. doi:10.1105/tpc. 108.061010

Brunet A, Park J, Tran H et al (2001) Protein kinase SGK mediates survival signals by phosphorylating the forkhead transcription factor FKHRL1 (FOXO3a). Mol Cell Biol 21:952-965. doi: 10.1128/MCB.21.3.952-965.2001

Burgering BM, Coffer PJ (1995) Protein kinase B (c-Akt) in phosphatidylinositol-3-OH kinase signal transduction. Nature 376:599-602. doi:10.1038/376599a0

Burgering BMT, Kops GJPL (2002) Cell cycle and death control: long live Forkheads. Trends Biochem Sci 27:352-360. doi: 10.1016/S0968-0004(02)02113-8

Burnett PE, Barrow RK, Cohen NA et al (1998) RAFT1 phosphorylation of the translational regulators p70 S6 kinase and 4E-BP1. Proc Natl Acad Sci USA 95:1432-1437. doi:10.1073/pnas. 95.4.1432

Carrière A, Cargnello M, Julien L-A et al (2008) Oncogenic MAPK signaling stimulates mTORC1 activity by promoting RSKmediated raptor phosphorylation. Current Biol 18:1269-1277. doi:10.1016/j.cub.2008.07.078

Casamayor A, Torrance PD, Kobayashi T et al (1999) Functional counterparts of mammalian protein kinases PDK1 and SGK in budding yeast. Curr Biol 9:186-S4. doi:10.1016/S0960-9822 (99)80088-8

Chen B-R, Runge KW (2009) A new Schizosaccharomyces pombe chronological lifespan assay reveals that caloric restriction promotes efficient cell cycle exit and extends longevity. Exp Gerontol 44:493-502. doi:10.1016/j.exger.2009.04.004

Colicelli J (2004) Human RAS superfamily proteins and related GTPases. Science's STKE: signal transduction knowledge environment 2004:RE13. doi:10.1126/stke.2502004re13

Cybulski N, Hall MN (2009) TOR complex 2: a signaling pathway of its own. Trends Biochem Sci 34:620-627. doi:10.1016/j.tibs. 2009.09.004
Dan HC, Sun M, Yang L et al (2002) Phosphatidylinositol 3-kinase/ Akt pathway regulates tuberous sclerosis tumor suppressor complex by phosphorylation of tuberin. J Biol Chem 277: 35364-35370. doi:10.1074/jbc.M205838200

Deprost D, Yao L, Sormani R et al (2007) The Arabidopsis TOR kinase links plant growth, yield, stress resistance and mRNA translation. EMBO Rep 8:864-870. doi:10.1038/sj.embor. 7401043

Díaz-Troya S, Pérez-Pérez ME, Florencio FJ, Crespo JL (2008) The role of TOR in autophagy regulation from yeast to plants and mammals. Autophagy 4:851-865

Eddy SR (1998) Profile hidden Markov models. Bioinformatics 14:755-763. doi:10.1093/bioinformatics/14.9.755

Engelman JA, Luo J, Cantley LC (2006) The evolution of phosphatidylinositol 3-kinases as regulators of growth and metabolism. Nat Rev Genetics 7:606-619. doi:10.1038/nrg1879

Findlay GM, Harrington LS, Lamb RF (2005) TSC1-2 tumour suppressor and regulation of mTOR signalling: linking cell growth and proliferation? Curr Opin Genet Dev 15:69-76. doi: 10.1016/j.gde.2004.11.002

Finn RD, Tate J, Mistry J et al (2008) The Pfam protein families database. Nucleic Acids Res 36:D281-D288. doi:10.1093/nar/ gkm960

Fritz-Laylin LK, Assaf ZJ, Chen S, Cande WZ (2010) Naegleria gruberi de novo basal body assembly occurs via stepwise incorporation of conserved proteins. Eukaryot Cell 9:860-865. doi:10.1128/EC.00381-09

Goberdhan DCI, Ogmundsdóttir MH, Kazi S et al (2009) Amino acid sensing and mTOR regulation: inside or out? Biochem Soc Trans 37:248-252. doi:10.1042/BST0370248

Hall MN (2008) mTOR-what does it do? Transpl Proc 40:S5-S8. doi: 10.1016/j.transproceed.2008.10.009

Harrington LS, Findlay GM, Gray A et al (2004) The TSC1-2 tumor suppressor controls insulin-PI3K signaling via regulation of IRS proteins. J Cell Biol 166:213-223. doi:10.1083/jcb.200403069

Hartmuth S, Petersen J (2009) Fission yeast Tor1 functions as part of TORC1 to control mitotic entry through the stress MAPK pathway following nutrient stress. J Cell Sci 122:1737-1746. doi: $10.1242 /$ jcs.049387

Howe K, Bateman A, Durbin R (2002) QuickTree: building huge neighbour-joining trees of protein sequences. Bioinformatics 18:1546-1547. doi:10.1093/bioinformatics/18.11.1546

Hresko RC, Mueckler M (2005) mTOR.RICTOR is the Ser473 kinase for Akt/protein kinase B in 3T3-L1 adipocytes. J Biol Chem 280:40406-40416. doi:10.1074/jbc.M508361200

Hsu Y-C, Chern JJ, Cai Y et al (2007) Drosophila TCTP is essential for growth and proliferation through regulation of dRheb GTPase. Nature 445:785-788. doi:10.1038/nature05528

Hubbard TJ, Aken BL, Ayling S et al (2009) Ensembl 2009. Nucleic Acids Res 37:D690-D697. doi:10.1093/nar/gkn828

Huson D, Richter D, Rausch C et al (2007) Dendroscope: an interactive viewer for large phylogenetic trees. BMC Bioinforma 8:460. doi:10.1186/1471-2105-8-460

Ikeda K, Morigasaki S, Tatebe $\mathrm{H}$ et al (2008) Fission yeast TOR complex 2 activates the AGC-family Gad8 kinase essential for stress resistance and cell cycle control. Cell Cycle (Georgetown, Tex) 7:358-364

Inoki K, Li Y, Xu T, Guan K-L (2003) Rheb GTPase is a direct target of TSC2 GAP activity and regulates mTOR signaling. Genes Dev 17:1829-1834. doi:10.1101/gad.1110003

Inoki K, Ouyang H, Zhu T et al (2006) TSC2 integrates Wnt and energy signals via a coordinated phosphorylation by AMPK and GSK3 to regulate cell growth. Cell 126:955-968. doi: 10.1016/j.cell.2006.06.055

Jacinto E, Lorberg A (2008) TOR regulation of AGC kinases in yeast and mammals. Biochem J 410:19-37. doi:10.1042/BJ20071518 
Jacinto E, Loewith R, Schmidt A et al (2004) Mammalian TOR complex 2 controls the actin cytoskeleton and is rapamycin insensitive. Nat Cell Biol 6:1122-1128. doi:10.1038/ncb1183

Jensen CJ (1999) 90-kDa ribosomal S6 kinase is phosphorylated and activated by 3-phosphoinositide-dependent protein kinase-1. J Biol Chem 274:27168-27176. doi:10.1074/jbc.274.38.27168

Jones KT, Greer ER, Pearce D, Ashrafi K (2009) Rictor/TORC2 regulates Caenorhabditis elegans fat storage, body size, and development through sgk-1. PLoS Biol 7:e60. doi:10.1371/ journal.pbio. 1000060

Katoh K, Misawa K, Kuma K, Miyata T (2002) MAFFT: a novel method for rapid multiple sequence alignment based on fast Fourier transform. Nucleic Acids Res 30:3059-3066. doi: 10.1093/nar/gkf436

Kim E, Goraksha-Hicks P, Li L et al (2008) Regulation of TORC1 by Rag GTPases in nutrient response. Nat Cell Biol 10:935-945. doi:10.1038/ncb1753

Kobayashi T, Cohen P (1999) Activation of serum- and glucocorticoidregulated protein kinase by agonists that activate phosphatidylinositide 3-kinase is mediated by 3-phosphoinositide-dependent protein kinase-1 (PDK1) and PDK2. Biochem J 339(Pt 2):319-328

Kockel L, Kerr KS, Melnick M et al (2010) Dynamic switch of negative feedback regulation in Drosophila Akt-TOR signaling. PLoS genetics 6:e1000990. doi:10.1371/journal.pgen.1000990

Loewith R, Jacinto E, Wullschleger S et al (2002) Two TOR complexes, only one of which is rapamycin sensitive, have distinct roles in cell growth control. Molecular Cell 10:457-468. doi:10.1016/S1097-2765(02)00636-6

Lorberg A, Hall MN (2004) TOR: the first 10 years. Curr Top Microbiol Immunol 279:1-18

Ma XM, Blenis J (2009) Molecular mechanisms of mTOR-mediated translational control. Nat Rev Mol Cell Biol 10:307-318. doi: $10.1038 / \mathrm{nrm} 2672$

Ma L, Chen Z, Erdjument-Bromage $\mathrm{H}$ et al (2005) Phosphorylation and functional inactivation of TSC2 by Erk implications for tuberous sclerosis and cancer pathogenesis. Cell 121:179-193. doi:10.1016/j.cell.2005.02.031

Mahfouz MM, Kim S, Delauney AJ, Verma DPS (2006) Arabidopsis TARGET OF RAPAMYCIN interacts with RAPTOR, which regulates the activity of S6 kinase in response to osmotic stress signals. Plant Cell 18:477-490. doi:10.1105/tpc.105.035931

Manning BD (2004) Balancing Akt with S6K: implications for both metabolic diseases and tumorigenesis. J Cell Biol 167:399-403. doi:10.1083/jcb.200408161

Matsumoto S, Bandyopadhyay A, Kwiatkowski DJ et al (2002) Role of the Tsc1-Tsc2 complex in signaling and transport across the cell membrane in the fission yeast Schizosaccharomyces pombe. Genetics 161:1053-1063

Menand B, Desnos T, Nussaume L et al (2002) Expression and disruption of the Arabidopsis TOR (target of rapamycin) gene. Proc Natl Acad Sci USA 99:6422-6427. doi:10.1073/pnas. 092141899

Menon S, Manning BD (2008) Common corruption of the mTOR signaling network in human tumors. Oncogene 27(Suppl 2):S43S51. doi:10.1038/onc. 2009.352

Mukai H (1995) Identification of Schizosaccharomyces pombe gene psk1+, encoding a novel putative serine/threonine protein kinase, whose mutation conferred resistance to phenylarsine oxide. Gene 166:155-159. doi:10.1016/0378-1119(95)00553-1

Oldham S, Hafen E (2003) Insulin/IGF and target of rapamycin signaling: a TOR de force in growth control. Trends Cell Biol 13:79-85. doi:10.1016/S0962-8924(02)00042-9

Otsubo Y, Yamamato M (2008) M TOR signaling in fission yeast. Crit Rev Biochem Mol Biol 43:277-283. doi:10.1080/1040923 0802254911
Park J, Leong ML, Buse P et al (1999) Serum and glucocorticoidinducible kinase (SGK) is a target of the PI 3-kinase-stimulated signaling pathway. EMBO J 18:3024-3033. doi:10.1093/emboj/ 18.11.3024

Potter CJ, Pedraza LG, Xu T (2002) Akt regulates growth by directly phosphorylating Tsc2. Nat Cell Biol 4:658-665. doi:10.1038/ ncb840

Pullen N, Dennis PB, Andjelkovic M et al (1998) Phosphorylation and activation of p70s6k by PDK1. Science (New York, NY) 279:707-710. doi:10.1126/science.279.5351.707

Rehmann H, Brüning M, Berghaus C et al (2008) Biochemical characterisation of TCTP questions its function as a guanine nucleotide exchange factor for Rheb. FEBS Lett 582:3005-3010. doi:10.1016/j.febslet.2008.07.057

Remm M, Storm CE, Sonnhammer EL (2001) Automatic clustering of orthologs and in-paralogs from pairwise species comparisons. J Mol Biol 314:1041-1052. doi:10.1006/jmbi.2000.5197

Roux PP, Ballif BA, Anjum R et al (2004) Tumor-promoting phorbol esters and activated Ras inactivate the tuberous sclerosis tumor suppressor complex via p90 ribosomal S6 kinase. Proc Natl Acad Sci USA 101:13489-13494. doi:10.1073/pnas.0405659101

Sancak Y, Peterson TR, Shaul YD et al (2008) The Rag GTPases bind raptor and mediate amino acid signaling to mTORC1. Science (New York, NY) 320:1496-1501. doi:10.1126/science.1157535

Sarbassov DD, Guertin DA, Ali SM, Sabatini DM (2005) Phosphorylation and regulation of Akt/PKB by the Rictor-mTOR complex. Science (New York, NY) 307:1098-1101. doi:10.1126/ science. 1106148

Serfontein J, Nisbet RER, Howe CJ, de Vries PJ (2010) Evolution of the TSC1/TSC2-TOR signaling pathway. Sci Signal 3:ra49. doi: 10.1126/scisignal.2000803

Shalchian-Tabrizi K, Minge MA, Espelund M et al (2008) Multigene phylogeny of choanozoa and the origin of animals. PloS one 3:e2098. doi:10.1371/journal.pone.0002098

Shaw RJ, Cantley LC (2006) Ras, PI(3)K and mTOR signalling controls tumour cell growth. Nature 441:424-430. doi:10.1038/ nature 04869

Shevchenko A, Roguev A, Schaft D et al (2008) Chromatin Central: towards the comparative proteome by accurate mapping of the yeast proteomic environment. Genome Biol 9:R167. doi: 10.1186/gb-2008-9-11-r167

Soukas AA, Kane EA, Carr CE et al (2009) Rictor/TORC2 regulates fat metabolism, feeding, growth, and life span in Caenorhabditis elegans. Genes Dev 23:496-511. doi:10.1101/gad.1775409

Soulard A, Cohen A, Hall MN (2009) TOR signaling in invertebrates. Curr Opin Cell Biol 21:825-836. doi:10.1016/j.ceb. 2009.08.007

Stamatakis A, Ludwig T, Meier H (2005) RAxML-III: a fast program for maximum likelihood-based inference of large phylogenetic trees. Bioinformatics 21:456-463. doi:10.1093/bioinformatics/ bti191

Stokoe D (1997) Dual role of phosphatidylinositol-3,4,5-trisphosphate in the activation of protein kinase B. Science 277:567-570. doi: 10.1126/science. 277.5325 .567

Tatebe H, Shiozaki K (2010) Rab small GTPase emerges as a regulator of TOR complex 2. Small GTPases 1:180-182. doi: 10.4161/sgtp.1.3.14936

Tee AR, Blenis J (2005) mTOR, translational control and human disease. Semin Cell Dev Biol 16:29-37. doi:10.1016/j.semcdb. 2004.11.005

Thomas G, Hall MN (1997) TOR signalling and control of cell growth. Curr Opin Cell Biol 9:782-787

Urano J (2000) The Saccharomyces cerevisiae Rheb G-protein is involved in regulating canavanine resistance and arginine uptake. J Biol Chem 275:11198-11206. doi:10.1074/jbc.275.15.11198 
Urban J, Soulard A, Huber A et al (2007) Sch9 is a major target of TORC1 in Saccharomyces cerevisiae. Mol cell 26:663-674. doi: 10.1016/j.molcel.2007.04.020

van Dam TJP, Rehmann H, Bos JL, Snel B (2009) Phylogeny of the CDC25 homology domain reveals rapid differentiation of Ras pathways between early animals and fungi. Cell Signal 21:1579-1585. doi:10.1016/j.cellsig.2009.06.004

van Dam TJP, Bos J, Snel B (2011) Evolution of the Ras-like small GTPases and their regulators. Small GTPases 2:4-16. doi: 10.4161/sgtp.2.1.15113

Van Dongen S (2008) Graph clustering via a discrete uncoupling process. SIAM J Matrix Anal Appl 30:121-141

Vernoud V, Horton AC, Yang Z, Nielsen E (2003) Analysis of the small GTPase gene superfamily of Arabidopsis. Plant Physiol 131:1191-1208. doi:10.1104/pp.013052

Wang X, Fonseca BD, Tang H et al (2008) Re-evaluating the roles of proposed modulators of mammalian target of rapamycin complex 1 (mTORC1) signaling. J Biol Chem 283:3048230492. doi: $10.1074 / j b c . M 803348200$

Wennerberg K, Rossman KL, Der CJ (2005) The Ras superfamily at a glance. J Cell Sci 118:843-846. doi:10.1242/jcs.01660

Wullschleger S, Loewith R, Hall MN (2006) TOR signaling in growth and metabolism. Cell 124:471-484. doi:10.1016/j.cell.2006. 01.016

Zhang Y, Gao X, Saucedo LJ et al (2003) Rheb is a direct target of the tuberous sclerosis tumour suppressor proteins. Nat Cell Biol 5:578-581. doi:10.1038/ncb999

Zinzalla V, Stracka D, Oppliger W, Hall MN (2011) Activation of mTORC2 by association with the ribosome. Cell 144:757-768. doi:10.1016/j.cell.2011.02.014

Zoncu R, Efeyan A, Sabatini DM (2011) mTOR: from growth signal integration to cancer, diabetes and ageing. Nat Rev Mol Cell Biol 12:21-35. doi:10.1038/nrm3025 